\title{
ORIGINAL
}

\section{ANISAKIASIS EN PESCADOS FRESCOS COMERCIALIZADOS EN EL NORTE DE CÓRDOBA}

\author{
Rafaela de la Torre Molina, José Pérez Aparicio, Manuel Hernández Bienes, Rafael Jurado Pérez, \\ Antonio Martínez Ruso y Emilio Morales Franco.
}

Servicio de Salud Pública. Área Sanituria Norte de Córdoba.

\section{RESUMEN}

Fundamento: La anisakiasis es una zomosis parasitaria producida por larvas vivas de nematodos de la Fanilia Anisakidae, que se localizan en la cavidad corporal y el sistema muscular de peces teleósteos y moluscos cefalópodos. El hombre las ingiere accidentalmente al consumir pescado infestade crudo o poco coxinkab. El cuadro clínico está caracterizado por sindronk gástico-intesinal. El objetivo del presente trabajo es conocer la frecuencia de parasitación por larsas de anisákidos en pescados frescos conkercializados en el note de la provincia de Córdoba.

Métodos: Durante un períndo de 9 neses se tomuron muestrus de las es-

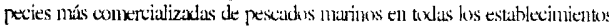
del sector. El análisis parasitológica se realizí en el Lahoratorio de Sadud Públic: de Peñanoya mediante el nétuxk) de la disección. Se analizann un total de 1.261 muesures de 18 especies distintas.

Resultados: La frecuencis total de parasitación fue del $15,8 \%$. La especie más parasitada fue la bacaladilla (Mirromesistias poutasou), con un porcentaje de parasitación de] $42 \%$, y se detectarno también larvas do Anisukidos en: pijota (Merlucius merlucius) un 27,5\%, pescudilla (Merlarias sp.) un 26\%. Caballa (Sromber scombras) un 20,6\%, brótula (Plycis blennoves) un $6,2 \%$, hoquerón (Engrualis encrasicolus) un $5,6 \%$ y faneco (Trisopterus lascus) un $5,5 \%$ de nuestras parasitudas, La distribución mensual de los resultados refleja un aumento de la parasitación en los meses de primavera. descendiendo progesivanxente hasta los últimos meses del an̂t.

Conclusiones: Existe una incidencia significativa de infestación pxor anisakidos en las especies de pexiolos connerciulizadas en el morte de Córdobit No obstante, la dificultad para detectar el parásitor, la valoración inperecisa del grado de parasitución tolerable o inaceptable que sejiala el marco nombativo, la cincunstancia de que algunos platos tradicionales elaborad s con pescado cn-

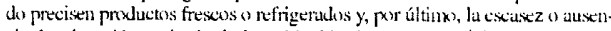
cia de educación sanitaria de la población, hacen aconscjable abondar este problemia emergente con mayor rigor.

Palabras clave: Parasitusis, Anisakiasis, Zoonosis, Pescados frescos.

\section{ABSTRACT \\ Anisakiasis in Fresh Fish Marketed in Northern Cordoba}

\begin{abstract}
Background: Anisikiasis is a parasitic zemmosis caused by live larvote of the Anischidas Fanily found lowated in the bonly cavities and in the nuscular systems of osseous fish and cephatopod mollusks. Homins inadvertently ingest these lanat when eating naw or undere $x$ ked infested fish. Symptons are characterized by gastrointestinal disonhers. The puppox of this study is that af axemtaining the frequency of anisukid larvate parasite infestation in tiesh tish marketed in northem section of the province of Cordoba.

Methods: Over a nine-munth periud, samples were taken of the species of sea fish sold to the greatest degree at all of the establislunkents in this sector: The parasite analysis was conducted at the Peñanya Public Health Laboratory by the dissection method. A tutal of 1,261 samples of 18 different species were analyzed.

Results: The total frequency of parasite infextation was $15.8 \%$. The species in which the nost pallasites were fordnd was the blue whiting (Micm)mesistios poutassou) slowing a $42 \%$ parasite infestition rate, and Anisikidos larvate were also found in: hake (Merlucius merlucius) (27.5\%), whiting (Merlucius sp.) (26\%), mackenel (Sromber srombrus), greater forkbeand (Phyris bimmoldes) (6.2\%), anclovy (Fngratis encravirohis) (5.6\%) and pouting (Trisopterus luscus) $(5.5 \%)$ of the samples in which purasites were found. The menthly spread of the results reveals an increase in parasite infestation during the spring nxontlis, deciming progressively up of the last month of of the year:

Conclusions: There is a significant degre of Anisakidos infestation in the species marketed in the norlen section of the province of Corduba. Nevertleless, the difliculty of detecting this parasite, the vague assessment of the degree of parasite infestation tolerable or unacceptable as set forth under regulations, the fact that some traditional dishes made from raw fish require fresh or nefrigerated products and, lastly, due lack or complete lack of health education on the part of the population thake it advisable for this emerging problem to be approuched witl greater rigor.
\end{abstract}

Key words: Parasitosis. Anisakiasis, Zoonosis. Fresh tish.
Correspondencia:

Rafaela de la Tome Molina

Distrito Sanitario de Pozoblanco

Ctru. Alcaracejos $s / n$

14400-Pozoblanco

Correo electrónico: mhbienes@hotmail.com

\section{INTRODUCCIÓN}

La anisakiasis es una zoonosis parasitaria producida por larvas vivas de nematodos de la Familia Anisakidae, que se localizan en la cavidad corporal o el sistema muscular de peces teleósteos y moluscos cefalópodos, y que son ingeridas accidentalmente por el 
hombre cuando consume pescado infectado crudo o poco cocinado (ahumados, adobo, salazones, etc.). El cuadro clínico se traduce en un síndrome gastrointestinal, oscilando desde una presentación leve con náuseas y dolor abdominal hasta una forma más intensa con sintomatología propia de apendicitis $u$ obstrucción intestinal. Aunque se han publicado trabajos señalando al parásito como una nueva fuente de antígenos alimentarios ${ }^{1}$ y responsable de reacciones alérgicas ${ }^{2}$, incluso se distinguió una nueva entidad clínica en la que los síntomas predominantes eran de hipersensibilidad $^{3-10}$, recientes investigaciones apuntan a una sola enfermedad, confirmando la tolerancia de enfermos a larvas no infestivas $^{11-13}$. El diagnóstico de la enfermedad es difícil y sólo da certeza la visualización de las larvas por gastroscopia, no obstante, el antecedente dietético, el cuadro y los hallazgos clínicos, así como exámenes alergológi$\cos ^{14}$, permiten establecer un diagnóstico de sospecha. La anisakiasis es frecuente en la población japonesa, en la que el hábito de consumir pescado crudo está muy extendido, mientras que en Europa y de manera especial en nuestro país, la preocupación por este problema ha ido creciendo en los últimos años ${ }^{15}$.

Las larvas de anisakidos pueden sobrevivir casi 2 meses en vinagre ${ }^{16}$ y se destruyen por el calor a $60^{\circ} \mathrm{C}$ durante 10 minutos o por el frío a $-20^{\circ} \mathrm{C}$ durante $3-5$ días. No se destruyen por tratamiento en el microondas ${ }^{17-24}$.

La infestación humana se ha asociado al consumo de platos tradicionales preparados con pescado crudo (sushi, sashimi, ceviche $)^{16,25}$ o ingestión de pescado crudo o ligeramente salado ${ }^{26}$. El primer caso de anisakiasis humana fue declarado por Straub en Holanda en $1955^{27}$. En España, los casos de Anisakiasis humana descritos hasta finales de 1998 han sido 32 , siendo Arenal Veral et al. ${ }^{28}$ quienes publican en 1991 el primer caso de esta enfermedad. Barros et al ${ }^{29}$ en 1992 publican tres casos de anisakiasis humana en España por consumo de sardinas crudas y, por último, López Peñas et $a^{27}$, describen 13 casos de anisakiasis en la provincia de Córdoba.
Dos de cstos casos ingresaron en el Hospital Valle de los Pedroches de Pozoblanco y los 13 enfermos manifestaron haber consumido platos tradicionales elaborados a partir de pescado crudo, principalmente boquerones en vinagre.

En España son escasas las investigaciones sobre la presencia de larvas de anisakidos en los productos pesqueros. No obstante se han detectado e identificado larvas de Anisakidos spp. en numerosos peces de nuestro litoral. Los servicios veterinarios de Córdoba $^{30}$ en el año 1982, detectaron en más de 100 ejemplares analizados, de 2 a 3 larvas de anisakis en la cavidad abdominal de bacaladillas ( $\mathrm{Mi}$ cromesistius poutassou). Olmedo y Berenguer ${ }^{31}$ en 1991, analizando 92 muestras de pescadilla fresca (Merlucius merlucius) recogidas en las comunidades autónomas de Castilla-La Mancha y Madrid, encuentran un índice de parasitación del 29,3\%. López Giménez y Castell ${ }^{32}$ en un estudio realizado también en Castilla $\mathrm{La}$ Mancha detectaron como especies más parasitadas, el congrio (Conger conger) con un $75 \%$, el jurel (Trachurus trachurus) y la caballa (Scomber scombrus) con un 42,1 y $32 \%$ respectivamente. No observaron larvas de anisakis en sardina (Sardina pilchardus), faneca (Trisopterus luscus), boquerón (Engraulis encrasicolus) y lenguado (Solea vulgaris) entre otros. En 1993, estos mismos autores detectan una parasitación media del $22,9 \%$, en muestras tomadas en las cinco provincias de Castilla La Mancha. Cuellar et $a^{33}$ diagnostican una parasitación que alcanza al $100 \%$ en triglas (Aspitrigla cuculus $100 \%$ y Eutrigla gurnardus $95,3 \%$ ), un $88 \%$ en merluza (Merlucius merlucius) y un $67 \%$ en caballa (Scomber scombrus). Por último, los resultados de un estudio realizado sobre 514 ejemplares de 20 especies de pescados de consumo habitual, recogidos en Merca-Bilbao, procedentes de diferentes puertos españoles, indicaron que, a excepción de la sardina (Sardina pilchardus), todas las especies examinadas estaban parasitadas por larvas de anisakidos. Sin embargo otros autores han señalado la presencia de larvas de anisakidos en sardinas capturadas en las costas europeas ${ }^{34}$. 
El objetivo del presente trabajo es conocer la frecuencia de parasitación por larvas de anisakidos en pescados frescos comercializadas en el norte de la provincia de Córdoba.

\section{MATERIAL Y MÉTODOS}

El norte de la provincia de Córdoba comprende 25 municipios que integran el Área Sanitaria Norte de Córdoba (ASNC), ordenados a efectos sanitarios en 4 Zonas Básicas de Salud (Z.B.S. de Pozoblanco, Peñarroya, Villanueva de Córdoba e Hinojosa del Duque) y un hospital de referencia (Hospital Comarcal Valle de los Pedroches).

Es una zona agrícola y pecuaria, con una población de 89.381 habitantes. El nivel de instrucción es bajo y la tasa de envejecimiento alta. Los indicadores económicos y de bienestar social son inferiores a la media nacional.

El censo de establecimientos del sector de productos de la pesca es de 4 industrias de almacenamiento y distribución de pescado fresco y 88 comercios de venta directa al consumidor (pescaderías).

Durante un periodo de nueve meses, realizamos tomas de muestras mensuales en todas las pescaderías de los municipios integrados en el Ârea Sanitaria Norte de Córdoba. La toma de muestras fue efectuada por los Veterinarios Oficiales adscritos al Servicio de Salud Pública, durante los meses de abril a diciembre de 1999. Cada muestra fue introducida en bolsa estéril y trasladada inmediatamente en neveras portátiles termoeléctricas al Laboratorio de Salud Pública de Peñarroya, donde se realizó el análisis parasitológico.

Tras la identificación morfológica de la especie de pescado, se utilizó el método de la disección para la investigación de las larvas, consistente en:

1. Incisión en la línea media ventral del pescado sin eviscerar, desde el espacio interopercular hasta el ano.
2. Extracción de las vísceras de la cavidad abdominal.

3. Examen visual de vísceras, cavidad abdominal, peritoneo y planos musculares adyacentes recogiendo todas las larvas visibles.

4. Además, en especies con piel fina (merluza y bacaladilla) se investigó la presencia de larvas en tejido subcutáneo.

5. Fileteado paralelo a la columna vertebral.

6. Examen visual de los planos musculares, recogiendo, así mismo, las larvas detectadas.

7. Identificación de las larvas aisladas mediante lupa binocular de 210 aumentos.

El número total de muestras recogidas fue de 1.288 , siendo investigadas un total de 1.261 muestras, pertenecientes a 17 especies diferentes de pescados marinos y 1 especie de moluscos cefalópodos. En la distribución del número de muestras por especie se observa que la bacaladilla (Micromesistius poutassou), sardina (Sardina pilchardus) y el boquerón (Engraulis encrasicolus) son las especies de las que se han tomado un mayor número de muestras, con 362, 294 y 269 respectivamente, ya que son las más habituales y consumidas. Les sigue el grupo de pijota (Merlucius merlucius) 58, faneca (Trisopterus luscus) 54, boga (Boops boops) 46, acedía (Dicologoglossa cuneata) 35, caballa (Scomber scombrus) 29, jurel (Trachurus trachurus) 27, pescadilla (Merlucius sp.) 23, brótola eviscerada (Phycis blennoides) $16 \mathrm{y}$ calamar (Loligo vulgaris) 15 muestras. Por último queda un grupo formado por especies que por su poca venta, el número de muestras ha sido muy escaso (tabla 1).

Los productos pesqueros comercializados en el norte de Córdoba proceden habitualmente de los caladeros del Golfo de Cádiz y Mediterráneo, y excepcionalmente del Atlántico. 
Tabla 1

Presencia de larvas de Anisakidos en Ias diferentes especies analizadas

\begin{tabular}{|c|c|c|c|}
\hline Especie & $N^{o}$ de muestras & $N^{o}$ de positivas & $\begin{array}{c}\text { Porcentaje de } \\
\text { positivas }\end{array}$ \\
\hline Bacaladilla (Micromesistius poutassou) & 362 & 152 & $42 \%$ \\
\hline Pijota (Merlucius merlucius) & 58 & 16 & $27,5 \%$ \\
\hline Pescadilla (Merlucius spp.) & 23 & 6 & $26 \%$ \\
\hline Caballa (Scomber scombrus) & 29 & 6 & $20,6 \%$ \\
\hline Brótola eviscerada (Phycis blennoides) & 16 & 1 & $6,2 \%$ \\
\hline Faneca (Trisopterus luscus) & 54 & 3 & $5,5 \%$ \\
\hline Boquerón (Engraulis encrasicolus) & 269 & 15 & $5,6 \%$ \\
\hline Sardina (Sardina pilchardus) & 294 & 0 & 0 \\
\hline Boga (Boops boops) & 46 & 0 & 0 \\
\hline Acedía (Dicologoglossa cuncata) & 35 & 0 & 0 \\
\hline Jurel (Trachurus trachurus) & 27 & 0 & 0 \\
\hline Aligote (Pagellus acarne) & 13 & 0 & 0 \\
\hline Salmonete de fango (Mullus barbatus) & 7 & 0 & 0 \\
\hline Lenguado (Solea vulgaris) & 4 & 0 & 0 \\
\hline Pejerrey (Atherina boyeri) & 4 & 0 & 0 \\
\hline Gallineta (Melicolenus dactylopterus) & 3 & 0 & 0 \\
\hline Rubio (Trigloporus lastoviza) & 2 & 0 & 0 \\
\hline Calamar (Loligo vulgaris) & 15 & 0 & 0 \\
\hline TOTAL & 1.261 & 199 & $15,8 \%$ \\
\hline
\end{tabular}

\section{RESULTADOS}

La frecuencia total de parasitación en las 1.261 muestras investigadas fue del $15,8 \%$ (Figura 1). Por Zonas Básicas de Salud el mayor porcentaje de parasitación lo presenta la de Villanueva de Córdoba $(23,2 \%)$ seguido de las de Pozoblanco (17,2\%), Peñarroya $(15,4 \%)$ y en menor porcentaje Hinojosa del Duque $(9,5 \%)$ (tabla 2$)$.
La cspecie más parasitada fue la bacaladilla (Micromesistius poutassou), con un porcentaje de parasitación del $42 \%$ (figura 2). Le sigue, pijota (Merlucius merlucius) con un $27,5 \%$, pescadilla (Merlucius sp.) con un $26 \%$, caballa (Scomber scombrus) con un 20,6\% brótola eviscerada (Phycis blennoides) con un $6,2 \%$, boquerón (Engraulis encrasicolus) con un 5,6\% y faneca (Trisopterus luscus) con un $5,5 \%$ (tabla 1). 
Figura 1

Porcentaje de parasitación por larvas de Anisakidos en el ASNC

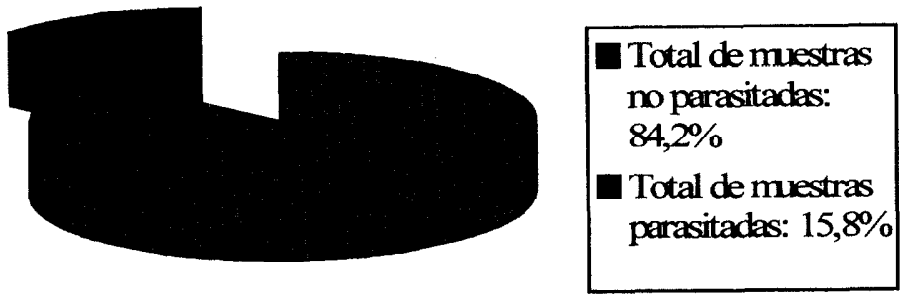

Figura 2

Porcentajes de parasitación por especies

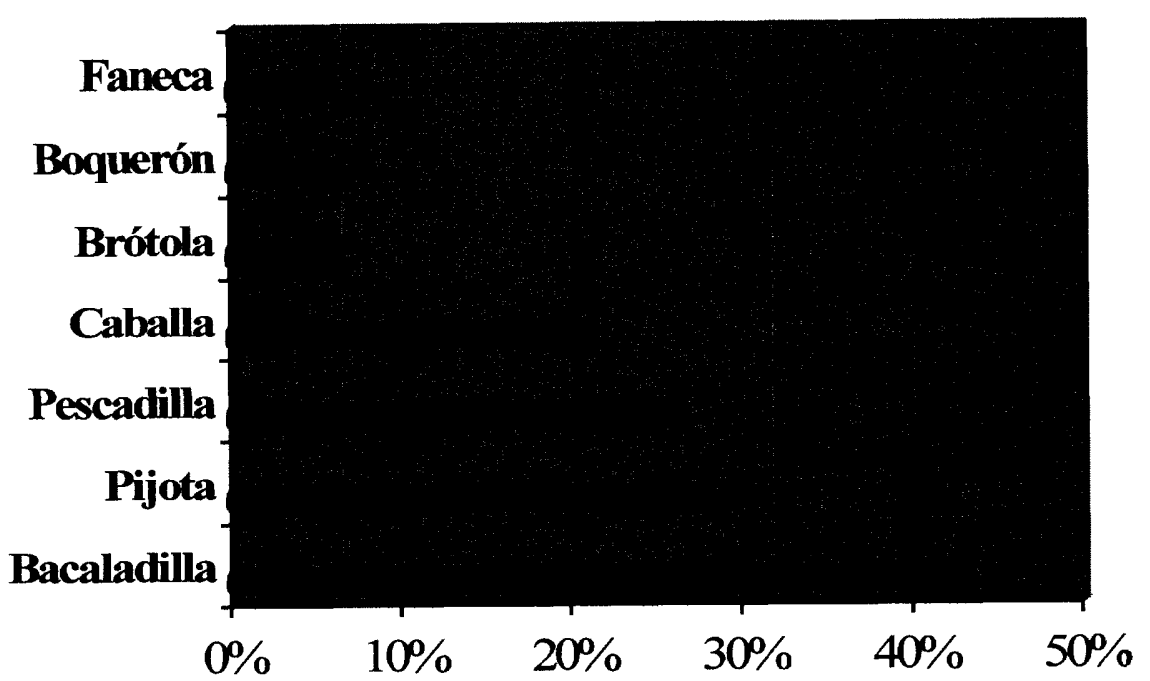

De las 18 cspecics diferentes estudiadas, en 11 de ellas no se ha detectado ninguna muestra parasitada. Clasificadas por orden de importancia comercial son: la sardina (Sardina pilchar$d u s$ ), boga (Boops boops), jurel (Trachurus trachurus), acedía (Dicologoglossa cuneata), y calamar (Loligo vulgaris), de las que se han analizado un total de 294, 46, 27, 35 y 15 muestras respectivamente sin ningún positivo. En el resto de especies en las que no se detectaron larvas de anisakidos, no podemos descartar parasitación ya que el número de muestras era muy escaso.

La distribución mensual de la tasa de parasitación no ha sido uniforme, presentando los meses de primavera (abril, mayo y junio) la mayor tasa, $26,3 \%, 32 \%$ y $28,2 \%$ respectivamente; disminuyendo en los meses de verano (julio, agosto y septiembre) con un $20,75 \%, 15 \%$ y $13,66 \%$ respectivamente y 
Tabla 1

Porcentaje de parasitación por larvas de Anisakidos por Z.B.S.

\begin{tabular}{|l|r|r|r|}
\hline \multicolumn{1}{|c|}{$Z B S$} & $N^{*}$ de muestras & $N^{*}$ de positivas & $\begin{array}{c}\text { Porcentaje de } \\
\text { positivas }\end{array}$ \\
\hline Peñarroya & 648 & 100 & $15,4 \%$ \\
\hline Hinojosa del Duque & 137 & 13 & $9,5 \%$ \\
\hline Pozoblanco & 407 & 70 & $17,2 \%$ \\
\hline Villanueva de Córdoba & 69 & 16 & $23,2 \%$ \\
\hline ASNC & 1.261 & 199 & $15,8 \%$ \\
\hline
\end{tabular}

en menor proporción los meses de otoño (octubre, noviembre y diciembre) con un $9 \%$, $6 \%$ y $5 \%$ respectivamente (Figura 3 ). Este hecho puede ser debido al elevado número de muestras y porcentaje de parasitación que presenta la bacaladilla (Micromesistius poutassou), que además de ser la especie más parasitada, es la única que presenta parasitación todos los meses estudiados. Las tasas más elevadas de esta especie se sitúan en los meses de primavera, para descender en los meses de verano y otoño $(66,6 \%, 87,5 \%$,
$59,5 \%, 63,6 \%, 36,4 \%, 40,4 \%, 16,2 \%, 13,5 \%$ y $18,4 \%$, respectivamente) (figura 4 ). Igual ocurre en la pijota (Merlucius merlucius) y la faneca (Trisopterus luscus), aunque el número de muestras procesadas por mes no es significativo. Sin embargo, no ocurre este hecho en el boquerón (Engraulis encrasicolus), especie con un número de muestras procesadas por mes significativo, ya que la mayor tasa de parasitación la presenta en los meses de primavera, seguida de los meses de otoño y en menor proporción, en los meses de verano.

Figura 3

Evolución mensual del total de muestras parasitadas

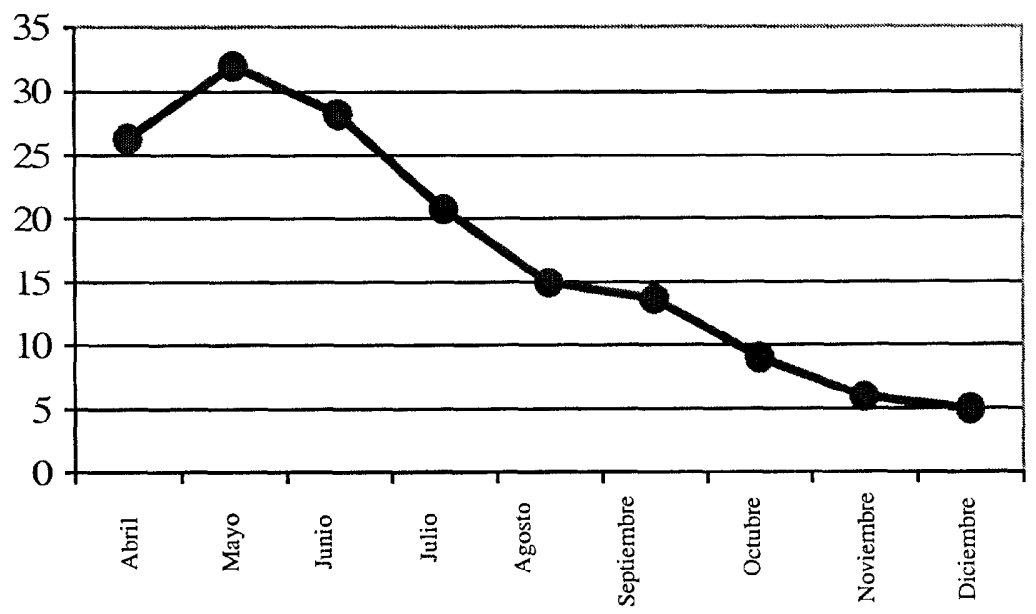


Figura 4

Evolución mensual de muestras de bacaladilla parasitadas

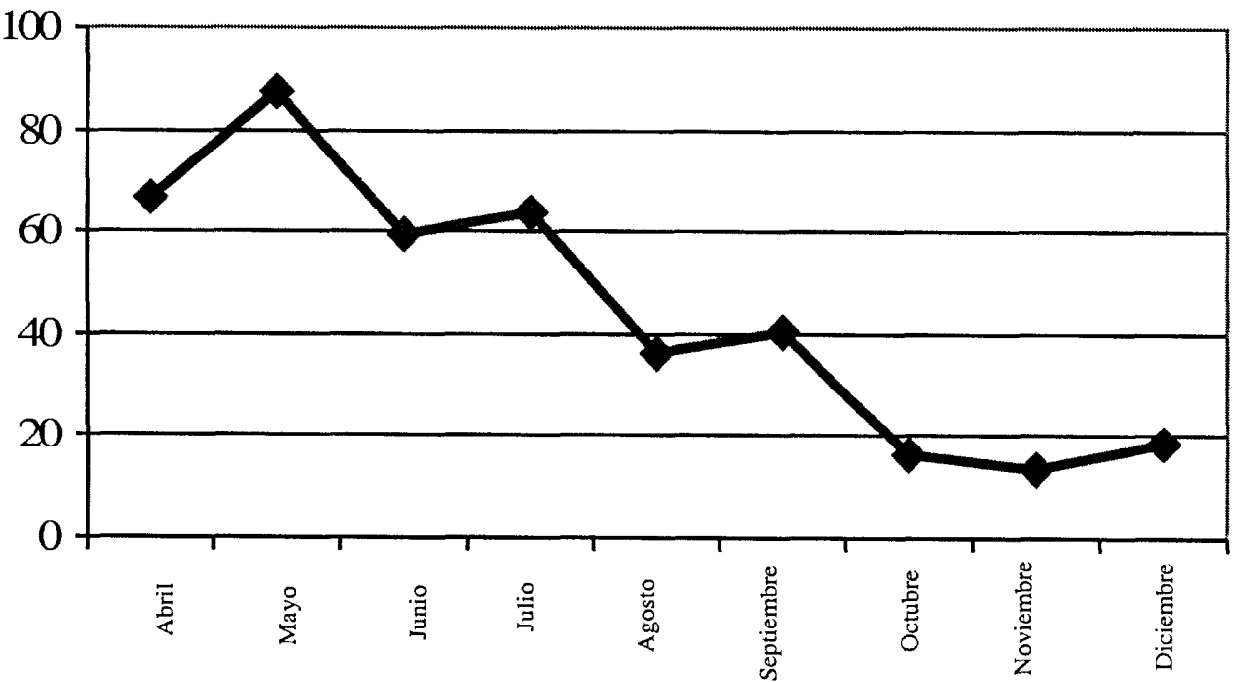

DISCUSIÓN

Los resultados muestran una incidencia significativa de infestación por anisakidos en las especies de pescados frescos comercializadas en el norte de la provincia de Córdoba y un grave riesgo sanitario, hecho confirmado por López Peñas et al ${ }^{27}$ en su estudio, al diagnosticar 13 casos de anisakiasis humana en la provincia de Córdoba. Dos de estos enfermos recibieron atención sanitaria en el Hospital Comarcal Valle de los Pedroches, hospital de referencia del ASNC.

En la clasificación de los resultados por ZBS no encontramos una relación significativa, y consideramos este hecho lógico ya que, aunque hay cuatro distribuidores distintos, la mayor parte de los productos proceden de las mismas lonjas del sur de nuestro país.

Hay que destacar también, el alto porcentaje de parasitación $(42 \%)$ que hemos detectado en la bacaladilla, una de las especies más comercializadas en el norte de Córdoba.
A diferencia de los resultados descritos por Pereira-Bueno ${ }^{34}$ en el estudio realizado en Merca-Bilbao, no hemos detectado parasitación por larvas de Anisakidos en boga (Boops boops), acedía (Dicologoglossa cuneata), jurel (Trachurus trachurus), aligote (Pagellus acarne) y salmonete de fango (Mullus barbatus). En el resto de especies en las que no se ha detectado larvas de anisakidos, el número de muestras fue muy escaso, y por ello no podemos descartar parasitación en este grupo. Tampoco detectamos en nuestro estudio parasitación en sardina (Sardina pilchardus) y sí detectamos parasitación en faneca (Trisopterus luscus) y boquerón (Engraulis encrasicolus) a diferencia de López Giménez y Castell ${ }^{32}$ que no encuentran parasitación en estas especies. Así mismo, no detectamos parasitación en jurel (Trachurus trachurus), a diferencia de López Giménez y Castell ${ }^{32}$ y Cuellar et $\mathrm{al}^{33}$.

Sí coincidimos con Pereira-Bueno ${ }^{34}$ al manifestar que la frecuencia de larvas en los peces puede variar en una misma zona a lo largo del tiempo, ya que durante el período 
de estudio hemos detectado diferencia entre los distintos meses.

Las prácticas de riesgo más comunes en nuestra zona son la elaboración de platos tradicionales a partir de pescados crudos, como los boquerones en vinagre, en el que sobreviven las larvas casi 2 meses $^{16}$, los preparados mediante asados, ya que el tratamiento térmico puede ser insuficiente y no uniforme $y$, por último, prácticas culinarias importadas como el ahumado.

Las investigaciones en productos pesqueros desarrolladas en España, segundo consumidor mundial de pescados por habitante, evidenciando una alta parasitación, así como el aumento de los casos de Anisakiasis comunicados han motivado una creciente preocupación y cuestionan la eficacia de las medidas existentes para garantizar la seguridad de estos productos.

La Directiva del Consejo 91/493/CEE, de 22 de julio traspuesta mediante el RD $1437 / 1992$, de 27 de noviembre ${ }^{35}$, establece que no podrán comercializarse los pescados o partes de pescado que presenten manifiestamente parásitos. Además, la Decisión de la Comisión de 19 de enero de $1993^{36}$ señala las modalidades de control visual para detectar parásitos en productos de la pesca. Por último, la legislación alimentaria ${ }^{37}$ también establece la obligatoriedad de congelar a -20 ${ }^{\circ} \mathrm{C}$ durante más de 24 horas todos los productos de la pesca destinados a consumirse crudos.

No obstante, la dificultad para detectar el parásito (control visual), la valoración imprecisa del grado de parasitación tolerable o inaceptable que señala el marco normativo (parasitación manifiesta), el desconocimiento a lo largo de toda la cadena alimentaria de la forma de consumo (crudo o cocinado), la circunstancia de que algunos platos tradicionales elaborados con pescado crudo precisen productos frescos o refrigerados (nunca congelados) y por último la escasez o ausencia de educación sanitaria a la población, hacen aconsejable abordar este problema emergente con mayor rigor.

\section{AGRADECIMIENTOS}

Expresamos nuestra gratitud a todos los Veterinarios Oficiales del Servicio de Salud Pública del Área Sanitaria Norte de Córdoba, así como al personal del Laboratorio de Salud Pública de Peñarroya por su colaboración inestimable.

\section{BIBLIOGRAFÍA}

1. Audicana M, Fernández de Corres L, Muñoz D, del Pozo MD, Fernández E et al. Anisakis simplex: una nueva fuente de antígenos alimentarios. Estudio de sensibilización a otros parásitos del orden Ascaridoidae. Rev Esp Alergol Immunol Clin 1995; 10: 325-31.

2. Daschnner A, Alonso Gómez A, Mora C, Moreno-Ancillo A, Villanueva $R$ y López-Serrano MC. Anisakiasis gastro-alérgica con parasitación masiva. Rev Esp Alergol Immunol Clin 1997; 12 (6): 370-2.

3. Daschner A, Alonso-Gómez A, Caballero T, Barranco P, Suarez de Parga JM y López Serrano MC. Gastric Anisakiasis: an underestimeted cause of acute urticaria and angioedema. $\mathrm{Br} \mathrm{J}$ Dermatol 1998; 139 (5): 822-8.

4. Audicana M, Fernández de Corres L, Muñoz D, Fernández E, Navarro JA y del Pozo MD. Recurrent anaphylaxis caused by anisakis simplex parasitizing fish. J Allergy Clin Immunol 1995; 96: 558-60.

5. Moreno-Ancillo A, Caballero MT, Cabañas R, Contreras J, Martín Barroso JA, Barranco P y López Serrano MC. Allergic reactions to Anisakis simplex parasitizing sea food. Ann allergy Asthma Immunol 1997; 79 : 246-50.

6. Alonso-Gómez A, Daschner A y Moreno-Ancillo A. Anaphylaxis with Anisakis simplex in gastric mucosa. N Engl J Med 1997; 337: 35051.

7. Daschnner A, Alonso Gómez A, Cabañas R, Suarcz de Parga JM y López Serrano MC. Gastroallergic anisakiasis: borderline between food allergy and parasitic disease: Clinical and aller- 
gologic evaluation of 20 patients with confirmed acute parasitism by Anisakis simplex. J Allergy Clin Immunol 2000; 105 (1): 176-81.

8. Del Pozo MD, Monen I, Fernández de Corres L, Audicana M, Muñoz D, Fernández E, Navarro JA y García M. Laboratory determinations in Anisakis simplex allergy. J Allergy Clin Immunol 1996; 97: 977-84.

9. Alonso-Gómez A, Daschner A, Moreno-Ancillo A, Barranco P, Suarez de Parga JM y López Serrano MC. Características clínica en Anisakiasis Gastroalérgica. Rev Port Immunoalergol 1997; 5 (2) (abstract 6).

10. López Serrano MC, Alonso-Gómez A, Daschner A, Moreno-Ancillo A, Suarez de Parga $\mathrm{JM}$, Caballero $\mathrm{T}$ et al. Gastro-allergy anisakiasis: findings in 22 patients. J Gastroenterol Hepatol 2000; 15 (5): 503-6.

11. López Serrano MC, Moreno-Ancillo A, Alonso Gómez A y Daschnner A. Anasakiasis in the year 2000. Rev Esp Enferm Dig 2000; 92 (3): $127-31$.

12. Domínguez Ortega J, Cimarra M, Sevilla MC, Alonso Llamazares A, Moneo I, Robledo Echarren $\mathrm{T}$ y Martínez-Cócera $\mathrm{C}$. Anisakis simplex: a cause of intestinal pseudo-obstrution. Rev Esp Enferm Dig 2000; 92 (3): 136-139.

13. Alonso A, Moreno-Ancillo A, Daschnner A y López-Serrano MC. Dietary assessment in five cases of allergic reactions due to gastroallergic anisakiasis. Allergy 1999; 54: 517-520.

14. Daschner A, Alonso-Gómez A, Caballero T, Suarez de Parga JM y López Serrano MC. Usefulness of early serial measurement of specific and total immunoglobulin $\mathrm{E}$ in the diagnosis of gastroallergic anisakiasis. Clin Exp Allergy 1999; 29 (9): 1260-4.

15. Presencia de Anisakis spp en pescados: repercusiones sanitarias y comerciales. Roma: Comisión del Codex Alimentarius, 2000. Documentos técnicos de la $22^{\circ}$ reunión dcl Comité Coordinador del Codex para Europa $00 / 10$.

16. Oshim T. Anisakiasis is the sushi bar guilty?. Parasitol Today 1987; 3: 44-48.

17. Fontaine RE. Anisakis from the American perspective. J Am Med Assoc 1985; 253: 10241025.
18. Bier JW, Decardorff TL, Jackson GJ y Raybour RB. Human anisakiasis. Bailleres Clin Trop Med 1987; 2: 723-733.

19. Lane CD, Master RN y Tietbohl RH. If your uneatenn food moves take it to a doctor. J Am Mcd Assoc 1988; 260: 340-341.

20. Bouree P, Paugam A y Petithory JC. Anisakidosis: report of 25 cases and review of the literature. Comp Immun Microbiol Infect Dis 1995; 18: $75-84$.

21. Louredo-Méndez A, Acedo de la Rosa F, Arribas de Paz V, Sanz Ortega E, Bernardo Quirós L y Goyanes Martínez A. Anisakidosis del colón como causa de abdomen agudo. Rev Esp Digest 1997; 89: 403-406.

22. Moreno-Ancillo A, Caballero MT, Cabañas R, Contreras J, Martín-Barroso JA, Barranco P et al. Allergic reactions to anisakis simplex parasitizing seafood. Ann Allergy Asthama Immunol 1997; 79: 246-250.

23. Del Olmo Escribano M, Cozar Ibáñez A, Martínez de Victoria JM y Ureña Tirao C. Anisakiasis a nivel ileal. Rev Esp Enferm Dig 1998; 90: 120-123.

24. Díaz Estruch J. Parasitosis por Nematodos. Alimentaria 1992 Junio; 43-45.

25. Sakanari JA y Mckerrow JH. Anisakiasis. Clin Microbiol Rev 1989; 2: 278-284.

26. Vieites Baptista de Sousa JM y Rodríguez Novoa MR. Parásitos más frecuentes en los productos de la Pesca: Su selección y visualización. Alimentaria 1992 Junio; 47-55.

27. López Peñas D, Ramírez Ortiz ML, del Rosal Palomeque R, López Rubio F, Fernández Crehuet R y Miño Fugarolas G. Estudio de 13 casos de anisakiasis en la provincia de Córdoba. Med Clin 2000; 114 (5): 177-180.

28. Arenal Vera JJ, Marcos Rodríguez JL, Borrego MH, Bowakin Did W, Castro Lorenzo J y Blanco Álvarez JL. Anisakiasis como causa de apendicitis aguda y cuadro reumatológico: El primer caso de la literatura médica. Rev Esp Enf Digest 1991; 79 (5): 355-358.

29. Barros C, Manzarbeitia F, López-Vélez R y Oñate JM. Anisakiasis Humana en España por consumo de sardinas crudas. Alimentaria 1992 Junio; 57-61.

30. Gómez LM. Aportación al estudio de nematosis (Anisakis spp. y contracaecum spp.) en la 
bacaladilla (Gadus poutassou) desde el punto de vista de la inspección veterinaria. Bol Inf del Cons Gral de Col Vet de España 1983; 29-31.

31. Olmedo Medicouague JR y Berenguer Soler J. Control de la incidencia de parasitación por nematodos del género anisakis sobre peces destinados al consumo humano. Inf Veterinaria 1991 Enero-febrero; 31-34.

32. López Giménez R y Castell Monsalve J. Estudio de la Tasa de Parasitación por Nematodos del Género Anisakis en el Pescado Fresco de venta más frecuente en Castilla La Mancha. Alimentaria 1994 Septiembre; 37-42.

33. Cuellar MC, Fontanillas JC, Pérez Fuentes J y Pérez Tauler MP. Biología y epidemiología de la anisakidosis larvaria. Enfermedad del arenque. En: Ciencias Veterinarias. Consejo Gral de Col Vet de España 1991; 4: 57-63.
34. Pereira-Bueno J. Algunos aspectos de la epidemiología y prevención de la anisakiasis. Valladolid: Consejería de Sanidad y Bienestar Social. Junta de Castilla y León; 1992.

35. Boletín Oficial del Estado. Real Decreto 1437/1992, de 27 de noviembre, por el que se fijan las normas sanitarias aplicables a la producción y comercialización de los productos pesqueros y de la acuicultura. BOE núm 11 , 21/1/1996.

36. Decisión de la Comisión de 19 de enero de 1993, por el que se establecen las modalidades del control visual para detectar productos de la pesca. DOCE, 9/3/1993.

37. ECOD. Directive 93/140EC. March 9, 1993, 156:42. 